\title{
Direction Finding with Gain/Phase Errors and Mutual Coupling Errors in the Presence of Auxiliary Sensors
}

\author{
Weijian Si, Di Wu, Lutao Liu, and Xinggen Qu \\ Department of Information and Communication Engineering, Harbin Engineering University, Harbin 150001, China \\ Correspondence should be addressed to Lutao Liu; liulutao@msn.com
}

Received 17 January 2014; Accepted 15 February 2014; Published 27 March 2014

Academic Editor: Shen Yin

Copyright (C) 2014 Weijian Si et al. This is an open access article distributed under the Creative Commons Attribution License, which permits unrestricted use, distribution, and reproduction in any medium, provided the original work is properly cited.

\begin{abstract}
Many classical direction of arrival (DOA) estimation algorithms suffer from sensitivity to array errors. A simple but efficient method is presented for direction finding in the presence of gain and phase errors as well as mutual coupling errors. By applying a group of auxiliary sensors, DOAs and gain and phase coefficients can be simultaneously estimated, and mutual coupling coefficients can also be estimated by utilizing a novel decoupling method. The proposed algorithm does not require iterative operation or any calibration sources or spectral peak searching. Simulation results demonstrate the effectiveness of the proposed method.
\end{abstract}

\section{Introduction}

The methods of the direction of arrival (DOA) estimation have received considerable attention because of their importance in a great variety of applications such as radar, sonar, and communication [1-5]. The subspace-based methods include multiple signal classification (MUSIC) algorithm [6] and estimation of signal parameters via rotational invariance techniques (ESPRIT) algorithm [7]. However, these high resolution methods rely on an exact characterization of the array manifold which is inevitably influenced by the array perturbations such as mutual coupling errors [8], gain and phase errors [9], and sensor position perturbation errors [10]. The performance of the direction finding methods will be greatly degraded with the array perturbations, and their applications are limited in practice. Therefore, it is essential to develop DOA estimation methods in the presence of array errors.

In recent decades, many researchers attempt to estimate the signal DOAs in the presence of array errors. To calibrate errors of mutual coupling, a number of calibration algorithms [11-18] have been proposed. A maximum-likelihood approach is developed in [11] by setting calibration sources with known locations. An alternative minimization procedure is presented in [12], but the multidimensional search of the associated nonlinear optimization is computationally complicated and convergence can not be guaranteed. DOAs and mutual coupling matrix are estimated in $[13,14]$ by utilizing rank-reduction (RARE) estimator based on the banded symmetric Toeplitz structural characteristics of the mutual coupling matrix. The methods developed in $[15,16]$ are based on auxiliary sensors for self-calibration of mutual coupling. Unfortunately, spectral peak search technique is required in these methods, which are limited in practical direction finding system because of multiple calculations. The methods in $[17,18]$ make use of auxiliary sensors similarly without requiring spectral peak search.

Besides researching the problem of mutual coupling errors, sensor gain and phase errors calibration methods [11, 19-25] have also been thoroughly investigated in the open literature. The active calibration method in [11] can also calibrate sensor gain and phase errors. The method in [19] can estimate the DOAs of signals and array parameters simultaneously based on the assumption that array perturbations are slight, but it suffers from suboptimal convergence caused by the joint iteration between DOA estimation and array parameter estimation. In [20], Liu et al. have proposed an eigenstructure DOA estimation method based on the eigendecomposition of a covariance matrix, which is constructed by dot product of array output and its conjugate. Shenghong et al. try to improve this eigenstructure DOA estimation method in [21]. However, at least two signals are spatially 
separated in the above two methods. In addition, the problem of DOA estimation using partially calibrated sensors or auxiliary sensors has been studied. The rank-reduction method [22-24] is typical, but it requires an additional spectral search. The root-RARE algorithm in [17] is computationally more efficient but also has its limitations. A simple and efficient method based on the conventional ESPRIT algorithm is proposed in [25], which can only calibrate the gain and phase errors.

In practical situations, various sources of array errors may be present simultaneously, but the aforesaid calibration methods are capable of analyzing statistical performance of circumstances where only one array error model presents. Assuming that sensor locations are known, array calibration can be considered as estimating the DOAs in the presence of sensor gain and phase errors and mutual coupling errors. Generally, calibration methods can be classified as active calibration methods [26-29] and self-calibration methods [30-33], which also have the above described problems.

In this paper, we propose a direction finding method in the presence of sensor gain and phase errors as well as mutual coupling errors. By applying a group of auxiliary sensors, we can eliminate mutual coupling errors by the inherent mechanism of the proposed method, assuming that DOA estimation is independent of mutual coupling coefficients. Then DOAs as well as gain and phase coefficients can be jointly estimated in closed form based on extended leastsquares ESPRIT (LS-ESPRIT) algorithm. Finally, mutual coupling coefficients can also be estimated by utilizing a new decoupling method. The proposed method requires no spectral search or iteration, so the complexity is low, which makes it simpler but more efficient, and it can realize the estimation of DOAs, gain/phase error coefficients, and mutual coupling coefficients simultaneously.

The rest of the paper is organized as follows. Section 2 illustrates problem formulation. In Section 3, the proposed method for estimation of DOA, sensor gain and phase error coefficients, and mutual coupling coefficients is described, and some discussions are given. Simulation results are presented in Section 4 to evaluate advantages of the proposed method over some representative calibration methods. At last, conclusions are drawn in Section 5.

\section{Problem Formulation}

Consider a uniform linear array (ULA) consisting of $M$ identical sensors with the space $d$ between neighboring sensors and $N$ uncorrelated narrowband far-field signals $s_{1}(t), s_{2}(t), \ldots, s_{N}(t)$ that arrive at the array from directions $\theta_{1}, \theta_{2}, \ldots, \theta_{N}$. Assuming that signals and noises are stationary, zero mean, uncorrelated random processes and noises are uncorrelated with variance $\sigma^{2}$; the output of the array is

$$
\mathbf{x}(t)=\mathbf{A s}(t)+\mathbf{n}(t),
$$

where $\mathbf{x}(t)$ is the $M \times 1$ array output vector, $\mathbf{x}(t)=$ $\left[x_{1}(t), x_{2}(t), \ldots, x_{M}(t)\right]^{\mathrm{T}}, \mathbf{s}(t)=\left[s_{1}(t), s_{2}(t), \ldots, s_{N}(t)\right]^{\mathrm{T}}$ is the $N \times 1$ vector of signal waveforms, and $\mathbf{n}(t)=$ $\left[n_{1}(t), n_{2}(t), \ldots, n_{M}(t)\right]^{\mathrm{T}}$ is the $M \times 1$ sensor noise vector that is commonly assumed to be the additive white Gaussian noise (AWGN). Moreover, $\mathbf{A}=\left[\mathbf{a}\left(\theta_{1}\right), \mathbf{a}\left(\theta_{2}\right), \ldots, \mathbf{a}\left(\theta_{N}\right)\right]$ is steering matrix in which the $i$ th signals direction manifold vector is denoted by $\mathbf{a}\left(\theta_{i}\right)=\left[1, \beta_{i}^{1}, \beta_{i}^{2}, \ldots, \beta_{i}^{N-1}\right]^{\mathrm{T}}$ with $\beta_{i}=$ $\exp \left\{-j 2 d \pi \sin \left(\theta_{i}\right) / \lambda\right\}$, and $i=1,2, \ldots, N$. $\lambda$ denotes carrier wavelength.

According to (1), the array covariance matrix of the array output is

$$
\mathbf{R}=E\left[\mathbf{x}(t) \mathbf{x}^{\mathrm{H}}(t)\right]=\mathbf{A} \mathbf{R}_{S} \mathbf{A}^{\mathrm{H}}+\sigma^{2} \mathbf{I},
$$

where $\mathbf{R}_{S}=E\left\{\mathbf{s}(t) \mathbf{s}^{\mathrm{H}}(t)\right\}$ is signal covariance matrix, $E\{\cdot\}$ represents the statistic expectation, and $\sigma^{2}$ and $I$ denote noise variance and $M \times M$ identity matrix, respectively.

The eigendecomposition of $\mathbf{R}$ is expressed as

$$
\mathbf{R}=\mathbf{U}_{S} \boldsymbol{\Sigma}_{S} \mathbf{U}_{S}^{\mathrm{H}}+\mathbf{U}_{V} \boldsymbol{\Sigma}_{V} \mathbf{U}_{V}^{\mathrm{H}},
$$

where $\mathbf{U}_{S}$ is signal subspace matrix containing eigenvectors with the largest eigenvalues and $\mathbf{U}_{V}$ is noise subspace matrix containing eigenvectors with the smallest eigenvalues. $\boldsymbol{\Sigma}_{S}$ and $\Sigma_{V}$ are diagonal matrices associated with signal power and noise power, respectively. In the case of finite snapshots, the array covariance matrix can be estimated as

$$
\widehat{\mathbf{R}}=\frac{1}{L} \sum_{i=1}^{L} \mathbf{x}(t) \mathbf{x}^{\mathrm{H}}(t),
$$

where $L$ is snapshot number. After the eigendecomposition of array covariance matrix is accomplished, signal subspace matrix $\widehat{\mathbf{U}}_{S}$ and noise subspace matrix $\widehat{\mathbf{U}}_{V}$ can be estimated.

Considering mutual coupling errors and gain and phase uncertainties of the array, the corresponding array output vector is rewritten by

$$
\mathbf{x}(t)=\Gamma \operatorname{CAs}(t)+\mathbf{n}(t),
$$

where $\mathbf{C}$ and $\boldsymbol{\Gamma}$ denote mutual coupling matrix (MCM) and gain and phase matrix (GPM), respectively, which are generally considered independent of direction.

As we know, mutual coupling coefficients of sensors are inversely related to their distance. On the basis of ULA structure, MCM can be expressed as a banded symmetric Toeplitz matrix. Suppose there are $p$ nonzero complex coefficients in MCM, with $p<M$. The $1 \times M$ coefficient vector can be written as

$$
\begin{aligned}
& \mathbf{c}=\left[c_{0}, c_{1}, \ldots, c_{p-1}, 0, \ldots, 0\right], \\
& 0<\left|c_{p-1}\right|<\left|c_{p-2}\right|<\cdots<c_{0}=1,
\end{aligned}
$$

where $c_{m}, m=1, \ldots, p-1$ is the mutual coupling coefficient. The corresponding $M \times M$ matrix $\mathbf{C}$ is given by

$$
\begin{aligned}
\mathbf{C} & =\operatorname{Toeplitz}(\mathbf{c}) \\
& =\text { Toeplitz }\left\{\left[1, c_{1}, \ldots, c_{p-1}, 0, \ldots, 0\right]\right\} .
\end{aligned}
$$

Actually, mutual coupling errors and gain and phase errors coexist. Assuming that the gain and phase of $M$ sensor 


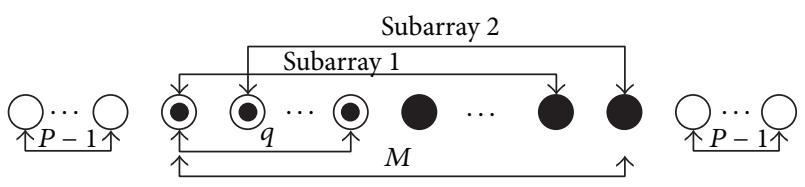

FIGURE 1: Array geometry. Solid points represent uncertain sensors and circles represent auxiliary sensors.

are uncertain, gain and phase coefficients can be presented by $\rho_{1}, \ldots, \rho_{M}$ and $\varphi_{1}, \ldots \varphi_{M}$, respectively. $M \times 1$ gain and phase vector is defined as

$$
\boldsymbol{\eta}=\left[\rho_{1} \mathrm{e}^{j \varphi_{1}}, \ldots, \rho_{M} \mathrm{e}^{j \varphi_{M}}\right]^{\mathrm{T}}
$$

and $M \times M G P M$ can be written as

$$
\Gamma=\operatorname{diag}\{\boldsymbol{\eta}\} .
$$

Here, we are interested in the problem of how to estimate the DOAs $\left\{\theta_{i}\right\}_{i=1}^{N}$ in the presence of sensors gain and phase errors and mutual coupling errors and how to obtain their coefficients, respectively.

\section{Proposed Method}

3.1. Array Model. Auxiliary sensors with known gain and phase are utilized to estimate DOAs, gain and phase coefficients, and mutual coupling coefficients on the basis of original $M$ sensors. First, we add $q$ sensors in front of $M$ sensors ULA and then add $p-1$ auxiliary sensors on each side of the $M+q$ sensors array. Thus a new array with a total of $M+q+2(p-1)$ sensors is formed, and the space between all neighboring sensors is $d$. Here $p$ is the mutual coupling degree of freedom without generality loss as mentioned above, which is assumed to be fixed. A detailed analysis will be performed in Section 3.5 concerning how the number of accurate calibration sensors $q$ affects algorithm performance. Besides, the array with a total of $M_{w}=M+$ $q+2(p-1)$ sensors is called whole array and that with $M_{m}=M+q$ middle sensors is called middle array.

Assuming that $q$ of sensors in the middle array are auxiliary sensors with known gain and phase, we can divide the middle array into two overlapping subarrays: one comprising first $M_{m}-1$ sensors where $q$ of them are well calibrated and the other comprising last $M_{m}-1$ sensors where $q-1$ of them are well calibrated. The array geometry is shown in Figure 1. Thus DOAs and gain and phase coefficients can be estimated by extended LS-ESPRIT algorithm which will be introduced below. Meanwhile, mutual coupling coefficients can be estimated by using the receiving data of the whole array.

Let $\overline{\mathbf{C}}$ and $\underline{\mathbf{C}}$ represent MCM of whole array and middle array, respectively. $\bar{\Gamma}$ and $\underline{\Gamma}$ denote their GPM, respectively. More precisely, gain and phase vectors are expressed as $\overline{\boldsymbol{\eta}}$ and $\boldsymbol{\eta}$, respectively.
We define matrix $\mathbf{P}$ as

$$
\mathbf{P}=\left[\begin{array}{lll}
\mathbf{0}_{M_{m} \times(p-1)} & \mathbf{I}_{M_{m} \times M_{m}} & \mathbf{0}_{M_{m} \times(p-1)}
\end{array}\right],
$$

where $\mathbf{0}_{M_{m} \times(p-1)}$ is an $M_{m} \times(p-1)$ zero matrix and $\mathbf{I}_{M_{m} \times M_{m}}$ is an $M_{m} \times M_{m}$ unit matrix. Thus the MCM $\overline{\mathbf{C}}$ of the whole array can be expressed as

$$
\overline{\mathbf{C}}=\left[\begin{array}{cccccc}
1 & c_{1} & \cdots & c_{p-1} & \cdots & 0 \\
c_{1} & 1 & c_{1} & \cdots & \ddots & 0 \\
\vdots & c_{1} & 1 & \ddots & \cdots & c_{p-1} \\
c_{p-1} & \cdots & \ddots & \ddots & c_{1} & \vdots \\
0 & \ddots & \cdots & c_{1} & 1 & c_{1} \\
0 & \cdots & c_{p-1} & \cdots & c_{1} & 1
\end{array}\right]_{M_{w} \times M_{w}}
$$

The MCM $\underline{\mathbf{C}}$ of the middle array is written by

$$
\begin{aligned}
\underline{\mathbf{C}} & =\mathbf{P} \overline{\mathbf{C}} \\
& =\left[\begin{array}{cccccccc}
c_{p-1} & \cdots & 1 & \cdots & c_{p-1} & 0 & \cdots & 0 \\
0 & c_{p-1} & \cdots & 1 & \cdots & c_{p-1} & \cdots & 0 \\
\vdots & \ddots & \ddots & \cdots & \ddots & \cdots & \ddots & \vdots \\
0 & \cdots & 0 & c_{p-1} & \cdots & 1 & \cdots & c_{p-1}
\end{array}\right]_{M_{m} \times M_{w}}
\end{aligned}
$$

Taking the unknown gain and phase uncertainties into account, their gain and phase vectors can be denoted by

$$
\begin{gathered}
\overline{\boldsymbol{\eta}}=\left[\mathbf{1}_{q+p-1}^{\mathrm{T}}, \rho_{1} \mathrm{e}^{j \varphi_{1}}, \ldots, \rho_{M} \mathrm{e}^{j \varphi_{M}}, \mathbf{1}_{p-1}^{\mathrm{T}}\right]^{\mathrm{T}}, \\
\underline{\boldsymbol{\eta}}=\left[\mathbf{1}_{q}^{\mathrm{T}}, \rho_{1} \mathrm{e}^{j \varphi_{1}}, \ldots, \rho_{M-q} \mathrm{e}^{j \varphi_{M}}\right]^{\mathrm{T}},
\end{gathered}
$$

respectively, where $\mathbf{1}$ is a vector with all elements equal to one. Here gain and phase matrix can be written as

$$
\begin{aligned}
& \overline{\boldsymbol{\Gamma}}=\operatorname{diag}\{\overline{\boldsymbol{\eta}}\}, \\
& \underline{\boldsymbol{\Gamma}}=\operatorname{diag}\{\underline{\boldsymbol{\eta}}\} .
\end{aligned}
$$

3.2. Joint Estimation of DOA and Gain and Phase Coefficient. Considering auxiliary sensors, the output of the middle array can be expressed as

$$
\underline{\mathbf{x}}(t)=\underline{\Gamma} \underline{\mathbf{C A}} \mathbf{s}(t)+\mathbf{n}(t),
$$

where $\overline{\mathbf{A}}=\left[\overline{\mathbf{a}}\left(\theta_{1}\right), \overline{\mathbf{a}}\left(\theta_{2}\right), \ldots, \overline{\mathbf{a}}\left(\theta_{N}\right)\right], \quad \overline{\mathbf{a}}(\theta)=$ $\left[1, \beta^{1}, \beta^{2}, \ldots, \beta^{M_{w}-1}\right]^{\mathrm{T}}$, and $M_{w}=M_{m}+2(p-1)$. Consequently, the steering matrix is given by 


$$
\begin{aligned}
\underline{\boldsymbol{\Gamma}} \underline{\mathbf{C}} \overline{\mathbf{a}}(\theta) & =\underline{\boldsymbol{\Gamma}}\left[\begin{array}{cccccccc}
c_{p-1} & \cdots & 1 & \cdots & c_{p-1} & 0 & \cdots & 0 \\
0 & c_{p-1} & \cdots & 1 & \cdots & c_{p-1} & \cdots & 0 \\
\vdots & \ddots & \ddots & \cdots & \ddots & \cdots & \ddots & \vdots \\
0 & \cdots & 0 & c_{p-1} & \cdots & 1 & \cdots & c_{p-1}
\end{array}\right]\left[\begin{array}{c}
1 \\
\beta \\
\vdots \\
\beta^{M_{m}+2 p-3}
\end{array}\right] \\
& =\underline{\Gamma}\left[\begin{array}{c}
c_{p-1}+\cdots+c_{1} \beta^{p-2}+\beta^{p-1}+c_{1} \beta^{p}+\cdots+c_{p-1} \beta^{2 p-2} \\
c_{p-1} \beta+\cdots+c_{1} \beta^{p-1}+\beta^{p}+c_{1} \beta^{p+1}+\cdots+c_{p-1} \beta^{2 p-1} \\
\vdots \\
c_{p-1} \beta^{M_{m}-1}+\cdots+c_{1} \beta^{M_{m}+p-3}+\beta^{M_{m}+p-2}+c_{1} \beta^{M_{m}+p-1}+\cdots+c_{p} \beta^{M_{m}+2 p-3}
\end{array}\right] \\
& \underline{\Gamma}\left[\begin{array}{c}
1 \\
\beta \\
\vdots \\
\beta^{M_{m}-1}
\end{array}\right] .
\end{aligned}
$$

Substituting $\beta=\exp \left\{-j 2 d \pi \sin \left(\theta_{i}\right) / \lambda\right\}$ into (17), we have

$$
\begin{aligned}
\underline{\boldsymbol{\Gamma}} \underline{\mathbf{C}} \overline{\mathbf{a}}(\theta)= & \beta^{p-1}\left(2 \sum_{m=1}^{p-1} c_{m} \cos \left(\frac{2 n \pi d \sin \theta}{\lambda}\right)+1\right) \\
& \times\left[\begin{array}{c}
1 \\
\beta \\
\vdots \\
\beta^{M_{m}-1}
\end{array}\right] \\
= & \underline{\boldsymbol{\Gamma} F} \underline{\mathbf{a}}(\theta),
\end{aligned}
$$

where $F=\beta^{p-1}\left(2 \sum_{m=1}^{p-1} c_{m} \cos (2 n \pi d \sin \theta / \lambda)+1\right)$ is a scalar function related to mutual coupling coefficient $c_{m}$ and the direction $\theta$ and $\underline{\mathbf{a}}(\theta)=\left[1, \beta, \ldots, \beta^{M_{m}-1}\right]$ is the ideal steering vector of the middle array.

According to the above analysis, MCM in the steering vector of the middle array is converted to the scalar function related to mutual coupling coefficients, thus realizing decoupling. Therefore, the steering vectors of two subarrays of the middle array are given by

$$
\begin{aligned}
& \widetilde{\mathbf{A}}_{1}=\Gamma_{1} F \mathbf{A}_{1}, \\
& \widetilde{\mathbf{A}}_{2}=\Gamma_{2} F \mathbf{A}_{2},
\end{aligned}
$$

where $\mathbf{A}_{1}$ and $\mathbf{A}_{2}$ denote the ideal steering vectors of these two subarrays, respectively, and $\Gamma_{1}$ and $\Gamma_{2}$ are GPM of these two subarrays, respectively, which can be written as

$$
\begin{aligned}
& \boldsymbol{\Gamma}_{1}=\operatorname{diag}\left\{\mathbf{1}_{q}^{\mathrm{T}}, \rho_{1} \mathrm{e}^{j \varphi_{1}}, \ldots, \rho_{M-q-1} \mathrm{e}^{j \varphi_{M_{m}-1}}\right\}, \\
& \boldsymbol{\Gamma}_{2}=\operatorname{diag}\left\{\mathbf{1}_{q-1}^{\mathrm{T}}, \rho_{1} \mathrm{e}^{j \varphi_{1}}, \ldots, \rho_{M-q} \mathrm{e}^{j \varphi_{M_{m}}}\right\} .
\end{aligned}
$$

The relationship between $\mathbf{A}_{1}$ and $\mathbf{A}_{2}$ is

$$
\mathbf{A}_{2}=\mathbf{A}_{1} \Phi
$$

where $\boldsymbol{\Phi}=\operatorname{diag}\left\{\mathrm{e}^{j 2 \pi d \sin \theta_{1} / \lambda}, \mathrm{e}^{j 2 \pi d \sin \theta_{2} / \lambda}, \ldots, \mathrm{e}^{j 2 \pi d \sin \theta_{N} / \lambda}\right\}$.
In the case that gain and phase errors and mutual coupling errors exist simultaneously, the received data of two subarrays are stacked into a column

$$
\begin{aligned}
\widetilde{\mathbf{x}}(t) & =\left[\begin{array}{l}
\mathbf{x}_{1}(t) \\
\mathbf{x}_{2}(t)
\end{array}\right]=\left[\begin{array}{l}
\Gamma_{1} F \mathbf{A}_{1} \\
\Gamma_{2} F \mathbf{A}_{2}
\end{array}\right] \mathbf{s}(t)+\mathbf{n}(t) \\
& =\widetilde{\mathbf{A}} \mathbf{s}(t)+\mathbf{n}(t),
\end{aligned}
$$

where $\widetilde{\mathbf{A}}=\left[\begin{array}{l}\boldsymbol{\Gamma}_{1} F \mathbf{A}_{1} \\ \boldsymbol{\Gamma}_{2} F \mathbf{A}_{2}\end{array}\right]$. Then the covariance matrix $\widetilde{\mathbf{R}}=$ $E\left[\widetilde{\mathbf{x}}(t) \widetilde{\mathbf{x}}^{\mathrm{H}}(t)\right]$ can be obtained. By taking the eigendecomposition of $\widetilde{\mathbf{R}}$, we arrive at the following equation:

$$
\widetilde{\mathbf{R}}=\widetilde{\mathbf{U}}_{S} \widetilde{\boldsymbol{\Sigma}}_{S} \widetilde{\mathbf{U}}_{S}^{\mathrm{H}}+\widetilde{\mathbf{U}}_{V} \widetilde{\boldsymbol{\Sigma}}_{V} \widetilde{\mathbf{U}}_{V}^{\mathrm{H}}
$$

where $\widetilde{\mathbf{U}}_{S}$ is the signal subspace spanned by eigenvectors corresponding to the big eigenvalues and $\widetilde{\mathbf{U}}_{V}$ is the noise subspace spanned by eigenvectors corresponding to the small eigenvalues; $\widetilde{\Sigma}_{S}$ and $\widetilde{\Sigma}_{V}$ are the diagonal matrixes associated with signal power and noise power, respectively. Since the signal subspace spanned by eigenvectors corresponding to the big eigenvalues is equal to the signal subspace spanned by array manifold, we have

$$
\operatorname{span}\left\{\widetilde{\mathbf{U}}_{S}\right\}=\operatorname{span}\{\widetilde{\mathbf{A}}\} .
$$

As it is known, $N \times N$ unique nonsingular matrix $\mathbf{T}$ satisfies the following formula:

$$
\widetilde{\mathbf{U}}_{S}=\widetilde{\mathbf{A}} \mathbf{T} \text {. }
$$

Obviously, (25) can be applied to the two subarrays equally, so we can get

$$
\widetilde{\mathbf{U}}_{S}=\left[\begin{array}{c}
\mathbf{U}_{S_{1}} \\
\mathbf{U}_{S_{2}}
\end{array}\right]=\left[\begin{array}{l}
\Gamma_{1} F \mathbf{A}_{1} \mathbf{T} \\
\Gamma_{2} F \mathbf{A}_{2} \mathbf{T}
\end{array}\right]=\left[\begin{array}{c}
\Gamma_{1} F \mathbf{A}_{1} \mathbf{T} \\
\Gamma_{2} F \mathbf{A}_{1} \boldsymbol{\Phi} \mathbf{T}
\end{array}\right],
$$

where $\mathbf{U}_{S_{1}}$ and $\mathbf{U}_{S_{2}}$ are signal subspaces of the two subarrays, respectively. Based on (26), the relationship between their signal subspaces can be expressed as

$$
\Gamma_{1} \mathbf{U}_{S_{2}}=\Gamma_{2} \mathbf{U}_{S_{1}} \Psi
$$


where $\Psi=\mathbf{T}^{-1} \boldsymbol{\Phi} \mathbf{T}$. For clarity, we rewrite the formula above as

$$
\widetilde{\Gamma} \mathbf{U}_{S_{2}}=\mathbf{U}_{S_{1}} \Psi
$$

where $\widetilde{\boldsymbol{\Gamma}}=\boldsymbol{\Gamma}_{1} \boldsymbol{\Gamma}_{2}^{-1}$. Combining the expression of $\boldsymbol{\Gamma}_{1}$ and $\boldsymbol{\Gamma}_{2}$, we can get

$$
\widetilde{\boldsymbol{\Gamma}}=\operatorname{diag}\{\widetilde{\boldsymbol{\eta}}\}
$$

Thus $\widetilde{\boldsymbol{\eta}}$ can be expressed as

$$
\begin{gathered}
\widetilde{\boldsymbol{\eta}}=\left[\mathbf{1}_{q-1}^{\mathrm{T}}, \rho_{1}^{-1} \mathrm{e}^{-j \varphi_{1}}, \rho_{1} \rho_{2}^{-1} \mathrm{e}^{-j\left(\varphi_{2}-\varphi_{1}\right)}, \ldots,\right. \\
\left.\rho_{M-q-1} \rho_{M-q}^{-1} \mathrm{e}^{j\left(\varphi_{M-q}-\varphi_{M-q-1}\right)}\right]^{\mathrm{T}} .
\end{gathered}
$$

As it is known that $\Psi$ is the similarity matrix of $N \times N$ matrix $\Phi$, it is easy to see that diagonal elements of $\Phi$ are eigenvalues of $\Psi$ and every column of $\mathbf{T}$ is the eigenvector of the matrix $\Psi$. In this case, we only need to estimate the matrix $\Psi$, and then $N$ eigenvalues $e_{1}, e_{1} \ldots, e_{N}$ can be obtained with eigendecomposition. Finally, DOAs of incident signals can be estimated by

$$
\theta_{k}=\sin ^{-1}\left\{\frac{\lambda \arg \left(e_{k}\right)}{2 \pi d}\right\}
$$

Considering the finite sampling data, the two signal subspace $\widehat{\mathbf{U}}_{S_{1}}$ and $\widehat{\mathbf{U}}_{S_{2}}$ are easily estimated. According to LS-ESPRIT algorithm and based on (27), we only need to minimize the following function to estimate the DOAs:

$$
f_{d}=\min \left\|\widetilde{\boldsymbol{\Gamma}}_{S_{2}}-\widehat{\mathbf{U}}_{S_{1}} \Psi\right\|_{F}^{2}
$$

where $\|\cdot\|_{F}$ is the Frobenius norm. Now, $\widetilde{\boldsymbol{\Gamma}}$ and $\boldsymbol{\Psi}$ are still unknown. By letting $\Psi$ be a variable and minimizing the function above, we can obtain the least-squares solution

$$
\Psi=\left(\widehat{\mathbf{U}}_{S_{1}}^{\mathrm{H}} \widehat{\mathbf{U}}_{S_{1}}\right)^{-1} \widehat{\mathbf{U}}_{S_{1}}^{\mathrm{H}} \widetilde{\boldsymbol{\Gamma}}_{S_{2}}
$$

Substituting formula (33) into (32), (32) can be expressed as

$$
\begin{aligned}
\left\|\widetilde{\boldsymbol{\Gamma}}_{S_{S_{2}}}-\widehat{\mathbf{U}}_{S_{1}} \Psi\right\|_{F}^{2} & =\left\|\mathbf{K} \widetilde{\boldsymbol{\Gamma}} \widehat{\mathbf{U}}_{S_{2}}\right\|_{F}^{2} \\
& =\operatorname{trace}\left\{\widehat{\mathbf{U}}_{S_{2}}^{\mathrm{H}} \widetilde{\boldsymbol{\Gamma}}^{\mathrm{H}} \mathbf{K}^{\mathrm{H}} \mathbf{K} \widetilde{\boldsymbol{\Gamma}} \widehat{\mathbf{U}}_{S_{2}}\right\} \\
& =\operatorname{trace}\left\{\widehat{\mathbf{U}}_{S_{2}} \widehat{\mathbf{U}}_{S_{2}}^{\mathrm{H}} \widetilde{\boldsymbol{\Gamma}}^{\mathrm{H}} \mathbf{K} \widetilde{\boldsymbol{\Gamma}}\right\},
\end{aligned}
$$

where the $\left(M_{m}-1\right) \times\left(M_{m}-1\right)$ matrix $\mathbf{K}$ is

$$
\mathbf{K}=\mathbf{I}-\widehat{\mathbf{U}}_{S_{1}}\left(\widehat{\mathbf{U}}_{S_{1}}^{\mathrm{H}} \widehat{\mathbf{U}}_{S_{1}}\right)^{-1} \widehat{\mathbf{U}}_{S_{1}}^{\mathrm{H}}
$$

and $\mathbf{K}^{\mathrm{H}} \mathbf{K}=\mathbf{K}$. Substituting (29) into (34) and applying the conclusion proved in [34], we get the following equation:

$$
\operatorname{trace}\left\{\widehat{\mathbf{U}}_{S_{2}} \widehat{\mathbf{U}}_{S_{2}}^{\mathrm{H}} \widetilde{\boldsymbol{\Gamma}}^{\mathrm{H}} \mathbf{K} \widetilde{\boldsymbol{\Gamma}}\right\}=\widetilde{\boldsymbol{\eta}}^{\mathrm{H}}\left(\left(\widehat{\mathbf{U}}_{S_{2}} \widehat{\mathbf{U}}_{S_{2}}^{\mathrm{H}}\right)^{\mathrm{T}} \odot \mathbf{K}\right) \widetilde{\boldsymbol{\eta}} \text {, }
$$

where $\odot$ is the Hadamard product. Here we assume that

$$
\mathbf{Q}_{d}=\left(\widehat{\mathbf{U}}_{S_{2}} \widehat{\mathbf{U}}_{S_{2}}^{\mathrm{H}}\right)^{\mathrm{T}} \odot \mathbf{K} .
$$

In that way, $\left\|\widetilde{\boldsymbol{\Gamma}} \widehat{\mathbf{U}}_{S_{2}}-\widehat{\mathbf{U}}_{S_{1}} \Psi\right\|_{F}^{2}=\widetilde{\boldsymbol{\eta}}^{\mathrm{H}} \mathbf{Q}_{d} \widetilde{\boldsymbol{\eta}}$. According to (30), it can be known that front the $q-1$ elements of $\widetilde{\boldsymbol{\eta}}$ are defined as 1 , so without loss of generality, the $(M-1) \times(q-1)$ matrix $\boldsymbol{\varepsilon}_{d}$ can be used to constrain $\widetilde{\boldsymbol{\eta}}$ :

$$
\boldsymbol{\varepsilon}_{d}=\left[\begin{array}{c}
\mathbf{I}_{(q-1) \times(q-1)} \\
\mathbf{0}_{\left(M_{m}-q\right) \times\left(M_{m}-q\right)}
\end{array}\right] .
$$

Thus solving the problem by minimizing the objective function (32) is transformed into

$$
\begin{array}{ll}
\min _{\widetilde{\boldsymbol{\eta}}} & \widetilde{\boldsymbol{\eta}}^{\mathrm{H}} \mathbf{Q}_{d} \widetilde{\boldsymbol{\eta}} \\
\text { s.t. } & \boldsymbol{\varepsilon}_{d}^{\mathrm{H}} \widetilde{\boldsymbol{\eta}}=\mathbf{1}_{q-1} .
\end{array}
$$

It should be noted that $\mathbf{Q}_{d}$ is a singular matrix for infinite sample data. Actually, sample data is finite, which implies that $\mathbf{Q}_{d}$ is a nonsingular matrix; thus the inverse matrix of $\mathbf{Q}_{d}$ exists, and the inverse matrix of $\mathbf{Q}_{d}^{\mathrm{H}}$ also exists. This is the necessary condition for the Lagrange method, which means the Lagrange method can be applied to solve the problem in (39). In this case, we can derive the Lagrange function

$$
L\left(\widetilde{\boldsymbol{\eta}}^{\mathrm{H}}, \mu\right)=\widetilde{\boldsymbol{\eta}}^{\mathrm{H}} \mathbf{Q}_{d} \widetilde{\boldsymbol{\eta}}-\mu\left(\boldsymbol{\varepsilon}_{d}^{\mathrm{H}} \widetilde{\boldsymbol{\eta}}-\mathbf{1}_{q-1}\right)
$$

and define that

$$
\begin{aligned}
& \nabla_{\widetilde{\eta}} L(\widetilde{\boldsymbol{\eta}}, \mu)=0, \\
& \nabla_{\mu} L(\widetilde{\boldsymbol{\eta}}, \mu)=0,
\end{aligned}
$$

where $\nabla$ is differential operator. From (41), the equations can be expressed as follows:

$$
\begin{aligned}
\widetilde{\boldsymbol{\eta}}^{\mathrm{H}} \mathbf{Q}_{d}-\mu \boldsymbol{\varepsilon}_{d}^{\mathrm{H}} & =0, \\
-\boldsymbol{\varepsilon}_{d}^{\mathrm{H}} \widetilde{\boldsymbol{\eta}}+\mathbf{1}_{q-1} & =0 .
\end{aligned}
$$

We rewrite these equations into the following matrix form:

$$
\left[\begin{array}{cc}
\mathbf{Q}_{d}^{\mathrm{H}} & -\boldsymbol{\varepsilon}_{d} \\
-\boldsymbol{\varepsilon}_{d}^{\mathrm{H}} & \mathbf{0}
\end{array}\right]\left[\begin{array}{c}
\widetilde{\boldsymbol{\eta}} \\
\mu^{\mathrm{H}}
\end{array}\right]=\left[\begin{array}{c}
0 \\
-\mathbf{1}_{q-1}
\end{array}\right] .
$$

Then it can be seen that the coefficient matrix $\left[\begin{array}{cc}\mathbf{Q}_{d}^{\mathrm{H}} & -\boldsymbol{\varepsilon}_{d} \\ -\boldsymbol{\varepsilon}_{d}^{\mathrm{H}} & \mathbf{0}\end{array}\right]$ is the Lagrange matrix. Assuming that this matrix is the nonsingular matrix, the inverse matrix can be expressed as

$$
\left[\begin{array}{cc}
\mathbf{Q}_{d}^{\mathrm{H}} & -\boldsymbol{\varepsilon}_{d} \\
-\boldsymbol{\varepsilon}_{d}^{\mathrm{H}} & \mathbf{0}
\end{array}\right]^{-1}=\left[\begin{array}{cc}
\mathbf{J}^{\mathrm{H}} & -\mathbf{V} \\
-\mathbf{V}^{\mathrm{H}} & \mathbf{S}
\end{array}\right] .
$$

Therefore, according to the definition of the inverse matrix, we can get

$$
\left[\begin{array}{cc}
\mathbf{Q}_{d}^{\mathrm{H}} & -\boldsymbol{\varepsilon}_{d} \\
-\boldsymbol{\varepsilon}_{d}^{\mathrm{H}} & \mathbf{0}
\end{array}\right]\left[\begin{array}{cc}
\mathbf{J}^{\mathrm{H}} & -\mathbf{V} \\
-\mathbf{V}^{\mathrm{H}} & \mathbf{S}
\end{array}\right]=\mathbf{I}_{2 n} .
$$


The detailed expression can be written as

$$
\begin{gathered}
\mathbf{Q}_{d}^{\mathrm{H}} \mathbf{J}^{\mathrm{H}}+\boldsymbol{\varepsilon}_{d} \mathbf{V}^{\mathrm{H}}=\mathbf{I}_{n}, \\
-\boldsymbol{\varepsilon}_{d}^{\mathrm{H}} \mathbf{J}^{\mathrm{H}}=\mathbf{0}_{n}, \\
-\mathbf{Q}_{d}^{\mathrm{H}} \mathbf{V}-\boldsymbol{\varepsilon}_{d} \mathbf{S}=\mathbf{0}_{n}, \\
\boldsymbol{\varepsilon}_{d}^{\mathrm{H}} \mathbf{V}=\mathbf{I}_{n} .
\end{gathered}
$$

Then, the expressions of matrix $\mathbf{J}^{\mathrm{H}}, \mathbf{V}^{\mathrm{H}}$, and $\mathbf{S}$ can be obtained by

$$
\begin{gathered}
\mathbf{J}^{\mathrm{H}}=\mathbf{Q}_{d}^{-\mathrm{H}}-\mathbf{Q}_{d}^{-\mathrm{H}} \boldsymbol{\varepsilon}_{d}\left(\boldsymbol{\varepsilon}_{d}^{\mathrm{H}} \mathbf{Q}_{d}^{-\mathrm{H}} \boldsymbol{\varepsilon}_{d}\right)^{-1} \boldsymbol{\varepsilon}_{d}^{\mathrm{H}} \mathbf{Q}_{d}^{-\mathrm{H}}, \\
\mathbf{V}^{\mathrm{H}}=\left(\boldsymbol{\varepsilon}_{d}^{\mathrm{H}} \mathbf{Q}_{d}^{-\mathrm{H}} \boldsymbol{\varepsilon}_{d}\right)^{-1} \boldsymbol{\varepsilon}_{d}^{\mathrm{H}} \mathbf{Q}_{d}^{-\mathrm{H}}, \\
\mathbf{S}=-\left(\boldsymbol{\varepsilon}_{d}^{\mathrm{H}} \mathbf{Q}_{d}^{-\mathrm{H}} \boldsymbol{\varepsilon}_{d}\right)^{-1},
\end{gathered}
$$

where $\mathbf{Q}_{d}^{-\mathrm{H}}$ denotes the inverse matrix of $\mathbf{Q}_{d}^{\mathrm{H}}$. In the following formulas, $\mathbf{Q}_{d}^{-1}$ is the inverse matrix of $\mathbf{Q}_{d}$.

Based on (43), it can be inferred that

$$
\begin{aligned}
{\left[\begin{array}{c}
\tilde{\boldsymbol{\eta}} \\
\mu^{\mathrm{H}}
\end{array}\right] } & =\left[\begin{array}{cc}
\mathbf{Q}_{d}^{\mathrm{H}} & -\boldsymbol{\varepsilon}_{d} \\
-\boldsymbol{\varepsilon}_{d}^{\mathrm{H}} & \mathbf{0}
\end{array}\right]^{-1}\left[\begin{array}{c}
0 \\
-\mathbf{1}_{q-1}
\end{array}\right] \\
& =\left[\begin{array}{cc}
\mathbf{J}^{\mathrm{H}} & -\mathbf{V} \\
-\mathbf{V}^{\mathrm{H}} & \mathbf{S}
\end{array}\right]\left[\begin{array}{c}
0 \\
-\mathbf{1}_{q-1}
\end{array}\right] .
\end{aligned}
$$

Consequently, $\widehat{\widetilde{\boldsymbol{\eta}}}$ can be obtained by

$$
\widehat{\widetilde{\boldsymbol{\eta}}}=\frac{\mathbf{Q}_{d}^{-1} \boldsymbol{\varepsilon}_{d} \mathbf{1}_{q-1}}{\boldsymbol{\varepsilon}_{d}^{\mathrm{H}} \mathbf{Q}_{d}^{-1} \boldsymbol{\varepsilon}_{d}} .
$$

Now, substituting (49) into (29) and (33), $\widehat{\Psi}$ and its eigendecomposition can be obtained. According to (31), we can get the DOAs of signals $\widehat{\theta}_{1}, \widehat{\theta}_{2}, \ldots, \widehat{\theta}_{N}$.

Since $\widehat{\widetilde{\boldsymbol{\eta}}}$ has been calculated by the process above, based on the definition of (30), gain and phase coefficients can be obtained easily:

$$
\begin{gathered}
\widehat{\rho}_{k} \mathrm{e}^{j \widehat{\varphi}_{k}}=\left(\prod_{i=q-1}^{q-1+k} \widehat{\widetilde{\eta}}_{i}\right)^{-1}, \\
\widehat{\boldsymbol{\eta}}=\left[\widehat{\rho}_{1} \mathrm{e}^{j \widehat{\varphi}_{1}}, \ldots, \widehat{\rho}_{M-q} \mathrm{e}^{j \widehat{\varphi}_{M-q}}\right]^{\mathrm{T}}, \\
\widehat{\boldsymbol{\Gamma}}=\operatorname{diag}\{\widehat{\boldsymbol{\eta}}\} .
\end{gathered}
$$

Such a solution process is a joint estimation of DOA and gain and phase coefficients.

3.3. Mutual Coupling Coefficient Estimation. After obtaining DOAs of incident signals and gain and phase coefficients, we will discuss mutual coupling coefficients estimation. Here, the output of the whole array is used. For the whole array with all sensors $M_{w}$, the output data is $\overline{\mathbf{x}}(\mathbf{t})$; the corresponding covariance matrix is expressed as $\overline{\mathbf{R}}$; then the noise subspace can be obtained by the eigendecomposition of $\overline{\mathbf{R}}$. In addition, we notice that the estimation of gain and phase coefficients has been carried out based on (51); hence, all elements of $\overline{\boldsymbol{\eta}}$ are well known. Due to the banded symmetric Toeplitz structure of MCM $\overline{\mathbf{C}}$, the transformation matrix of MCM corresponding to each mutual coupling coefficient can be represented individually. In this case, the $\overline{\mathbf{C}}$ corresponding to the whole array can be expressed as

$$
\begin{gathered}
\overline{\mathbf{C}}=\sum_{m=0}^{p-1} c_{m} \mathbf{E}_{m}, \\
\mathbf{E}_{m}(i, j)=\delta\left(\overline{\mathbf{C}}(i, j)-c_{m}\right), \\
\delta(k)= \begin{cases}1, & k=0, \\
0, & \text { otherwise. }\end{cases}
\end{gathered}
$$

Now, the array manifold taking the new form of $\overline{\mathbf{C}}$ can be expressed by

$$
\begin{aligned}
\overline{\boldsymbol{\eta}} \overline{\mathbf{C}} \overline{\mathbf{a}}(\theta) & =\overline{\boldsymbol{\eta}} \sum_{m=0}^{p-1} c_{m} \mathbf{E}_{m} \overline{\mathbf{a}}(\theta)=\overline{\boldsymbol{\eta}} \sum_{m=1}^{p-1} c_{m} \mathbf{W}_{m}(\theta) \\
& =\overline{\boldsymbol{\eta}}\left[\mathbf{W}_{1}(\theta), \mathbf{W}_{2}(\theta), \ldots, \mathbf{W}_{p-1}(\theta)\right] \overline{\mathbf{c}} \\
& =\overline{\boldsymbol{\eta}} \mathbf{W}(\theta) \overline{\mathbf{c}},
\end{aligned}
$$

where $\overline{\mathbf{c}}=\left[c_{0}, c_{1}, \ldots, c_{p-1}\right]^{\mathrm{T}}, \mathbf{W}(\theta)$ is $M \times p$ transform matrix, and each element is defined by

$$
\begin{aligned}
W_{i, j}(\theta)= \begin{cases}a_{i} & j=1, \\
a_{i+j-1}+a_{i+1-j} & 2 \leq j \leq p,\end{cases} \\
\quad i \in(1, \ldots, M), j \in(1, \ldots, p),
\end{aligned}
$$

where

$$
\begin{gathered}
a_{0}=a_{M}=0 \\
a_{-1}=a_{M+1}=0 \\
\vdots \\
a_{-(p-1)}=a_{M+(p-1)}=0 .
\end{gathered}
$$

Based on the subspace theory, MCM can be obtained by minimizing $f_{c}$, which is shown by

$$
f_{c}=\min \left\|(\overline{\boldsymbol{\eta}} \overline{\mathbf{C}} \overline{\mathbf{a}}(\theta))^{\mathrm{H}} \widehat{\mathbf{U}}_{V}\right\|_{F}^{2} .
$$

By substituting (56) into (59), $f_{c}$ can be rewritten as

$$
\begin{aligned}
f_{c} & =\min \left\|(\overline{\boldsymbol{\eta}} \mathbf{W}(\theta) \overline{\mathbf{c}})^{\mathrm{H}} \widehat{\overline{\mathbf{U}}}_{V}\right\|^{2} \\
& =\min \overline{\mathbf{c}}^{\mathrm{H}} \mathbf{W}^{\mathrm{H}}(\theta) \overline{\boldsymbol{\eta}}^{\mathrm{H}} \widehat{\overline{\mathbf{U}}}_{V} \widehat{\overline{\mathbf{U}}}_{V}^{\mathrm{H}} \overline{\boldsymbol{\eta}} \mathbf{W}(\theta) \overline{\mathbf{c}} \\
& =\min \overline{\mathbf{c}}^{\mathrm{H}} \mathbf{Q}_{c} \overline{\mathbf{c}},
\end{aligned}
$$

where $\mathbf{Q}_{c}=\mathbf{W}^{\mathrm{H}}(\theta) \overline{\boldsymbol{\eta}}^{\mathrm{H}} \widehat{\overline{\mathbf{U}}}_{V} \widehat{\overline{\mathbf{U}}}_{V}^{\mathrm{H}} \overline{\boldsymbol{\eta}} \mathbf{W}(\theta)$. Please note $\mathbf{Q}_{c}$ is totally independent of the mutual coupling coefficient vector $\overline{\mathbf{c}}$. 
Since sample data is finite, $\mathbf{Q}_{d}$ is a nonsingular matrix with inverse matrix. Without loss of generality, the first element of $\overline{\mathbf{c}}$ is constrained to 1 ; namely, $\overline{\mathbf{c}} \boldsymbol{\varepsilon}=1$ and $\boldsymbol{\varepsilon}=[1,0, \ldots, 0]^{\mathrm{T}}$. Thus the problem of minimizing $f_{c}$ is transformed into a quadratic programming problem with linear constraints, which is given by

$$
\begin{array}{ll}
\min & \overline{\mathbf{c}}^{\mathrm{H}} \mathbf{Q}_{c} \overline{\mathbf{c}} \\
\text { s.t. } & \boldsymbol{\varepsilon}^{\mathrm{T}} \overline{\mathbf{c}}=1 .
\end{array}
$$

Through the same method as that in Section 3.2, mutual coupling coefficients can be obtained by

$$
\overline{\mathbf{c}}=\frac{\mathbf{Q}_{c}^{-1} \boldsymbol{\varepsilon}}{\boldsymbol{\varepsilon}^{\mathrm{T}} \mathbf{Q}_{c}^{-1} \boldsymbol{\varepsilon}}
$$

Thus mutual coupling coefficients have been estimated. This mutual coupling coefficients estimation method is applicable to any form of antenna array; just the transform matrix $\mathbf{W}(\theta)$ is different. From the estimation of DOAs, gain and phase coefficients, and mutual coupling coefficients we can see that DOAs as well as gain and phase coefficients can be jointly estimated independently of mutual coupling coefficients firstly; with the known DOAs as well as gain and phase coefficients, mutual coupling coefficients can be estimated. So the three estimations do not affect mutually.

3.4. Algorithm Steps. The estimation method of DOAs, sensor gain and phase coefficients, and mutual coupling coefficients can be summarized as follows.

(1) Assuming a ULA with $M+q+2(p+1)$ elements, where $q+2(p+1)$ elements are auxiliary sensors, we use output data vector $\hat{\overline{\mathbf{x}}}(t)$ of the whole array to compute the covariance $\widehat{\overline{\mathbf{R}}}$

$$
\widehat{\overline{\mathbf{R}}}=\frac{1}{L} \sum_{i=1}^{L} \widehat{\bar{x}}(t) \widehat{\bar{x}}^{\mathrm{H}}(t)
$$

and utilize the output data of the first subarray $\mathbf{x}_{1}(t)$ as well as the second subarray $\mathbf{x}_{2}(t)$ of the middle array to get the output vector $\widehat{\widetilde{\mathbf{x}}}(t)$ according to (22). Then covariance $\widehat{\widetilde{\mathbf{R}}}$ can be obtained:

$$
\widehat{\widetilde{\mathbf{R}}}=\frac{1}{L} \sum_{i=1}^{L} \widehat{\widetilde{x}}(t) \widehat{\widetilde{x}}^{\mathrm{H}}(t) .
$$

(2) Perform eigendecomposition of $\widehat{\widetilde{\mathbf{R}}}$ and obtain the signal subspaces $\widehat{\mathbf{U}}_{S_{1}}$ and $\widehat{\mathbf{U}}_{S_{2}}$; perform eigendecomposition of $\widehat{\overline{\mathbf{R}}}$ and obtain the noise subspaces $\widehat{\overline{\mathbf{U}}}_{V}$.

(3) Based on the extending LS-ESPRIT algorithm of this paper and (53), $\overline{\tilde{\boldsymbol{\eta}}}$ can be calculated. Based on the relationship between $\widehat{\widetilde{\tilde{\eta}}}$ and $\widehat{\widetilde{\Gamma}}, \widehat{\widetilde{\Gamma}}$ can be estimated by (29). Substituting $\widehat{\widetilde{\Gamma}}$ into (33), we can obtain $\widehat{\Psi}$. Then by performing eigendecomposition, according to (31), we can obtain the DOAs of signals $\widehat{\theta}_{1}, \widehat{\theta}_{2}, \ldots, \widehat{\theta}_{N}$.
(4) Gain and phase coefficients $\widehat{\rho}_{1}, \ldots, \widehat{\rho}_{M}$ and $\widehat{\varphi}_{1}, \ldots \widehat{\varphi}_{M}$ can be estimated by formula (50).

(5) Mutual coupling coefficients $1, \widehat{c}_{1}, \ldots, \widehat{c}_{p-1}$ can be estimated by utilizing (62).

3.5. Performance Analysis and Comparison. In this paper, we focus on the simple solution to tackle sensor gain and phase errors and mutual coupling errors. Based on derivation in previous sections, it can be seen that the proposed method is computationally efficient because no spectral search and iteration are required. The contention WF method in [24] needs iteration and circular process, and the method in $[10$, 17] makes use of spectral-RARE estimator and thus requires spectral search.

With detailed analysis, there are some necessary conditions worthy of attention. First, in order to make the LS-ESPRIT algorithm work, we must use auxiliary sensors, and the number of $q$ must be no less than two. The more the number of $q$ is, the better the proposed algorithm performs. On each side of the array, $p-1$ auxiliary sensors are added. The number of $p-1$ is decided by $p$ nonzero complex coefficients of MCM.

\section{Simulation Results}

In this section, we present several simulation results to illustrate the performance of the proposed method. In the following examples, we consider a ULA of 14 sensors with half wavelength spacing, and 9 of them are auxiliary sensors, where $q=5, p=3$, and $M=5$, namely, $M_{w}=$ 14 and $M_{m}=10$. The mutual coupling vector is set as $\overline{\mathbf{c}}=[1,0.4499+j 0.5362,0.2598-j 0.1500]^{\mathrm{T}}$, and the gain and phase vector of uncertain sensors is given by $\eta=\left[0.8 \mathrm{e}^{j \pi / 5}, 1.25 \mathrm{e}^{-j \pi / 3}, 1.53 \mathrm{e}^{-j \pi / 5}, 0.75 \mathrm{e}^{j \pi / 4}, 1.36 \mathrm{e}^{-j \pi / 10}\right]^{\mathrm{T}}$. The background noise is assumed to be AWGN and the signal-to-noise ratio (SNR) of the input signals is defined as $10 \log _{10}\left(\sigma_{s}^{2} / \sigma_{n}^{2}\right)$. The following measures are used to evaluate the performance: $\mathrm{RMSE}_{\mathrm{D}}$ (root mean squared error of DOA estimation), $\mathrm{RMSE}_{G}$ and $\mathrm{RMSE}_{P}$ (RMSE of gain and phase estimation), and $\mathrm{RMSE}_{\mathrm{MCR}}$ and $\mathrm{RMSE}_{\mathrm{MCI}}$ (RMSE of real part and imaginary part of mutual coupling coefficient estimation):

$$
\begin{aligned}
\mathrm{RMSE}_{\mathrm{DOA}} & =\sqrt{\frac{\sum_{i=1}^{K} \sum_{n=1}^{N}\left[\left(\theta_{n}-\widehat{\theta}_{n, i}\right)^{2}+\left(\varphi_{n}-\widehat{\varphi}_{n, i}\right)^{2}\right]}{N K}}, \\
\mathrm{RMSE}_{G} & =\sqrt{\frac{\sum_{i=1}^{K}\left(G_{m}-\widehat{G}_{m i}\right)^{2}}{\left\|G_{m}\right\| K}} \times 100 \%, \\
\operatorname{RMSE}_{P} & =\sqrt{\frac{\sum_{i=1}^{K}\left(P_{m}-\widehat{P}_{m i}\right)^{2}}{\left\|P_{m}\right\| K}} \times 100 \%,
\end{aligned}
$$




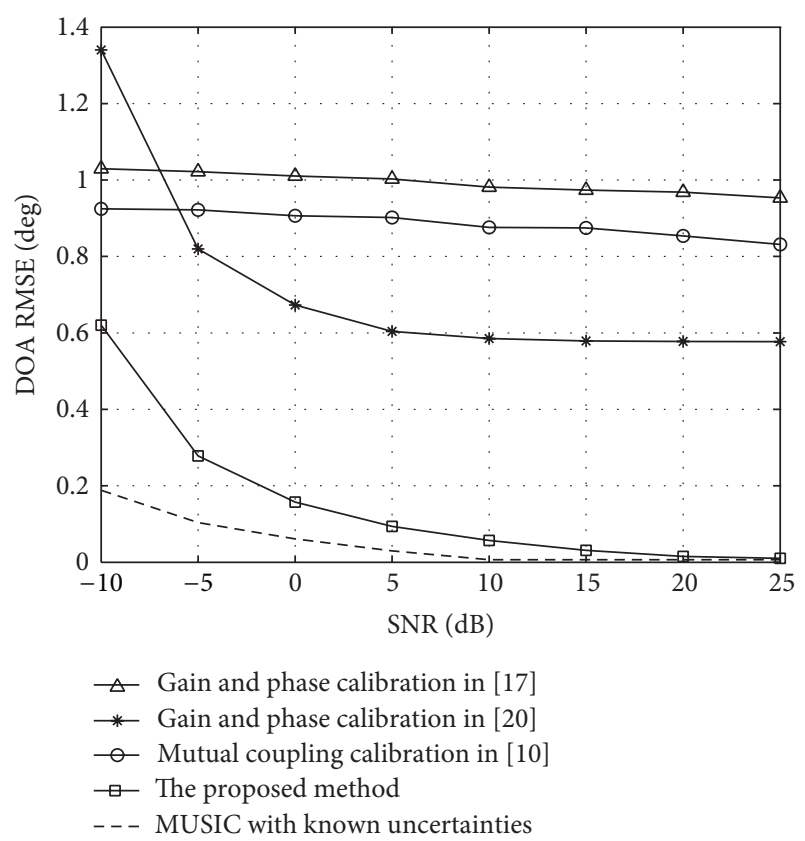

FIGURE 2: RMSE of two signals DOA estimation versus SNR.

$$
\begin{aligned}
\operatorname{RMSE}_{\mathrm{MCR}} & =\sqrt{\frac{\sum_{i=1}^{K}\left(R_{v}-\widehat{R}_{v i}\right)^{2}}{\left\|R_{v}\right\| K}} \times 100 \%, \\
\operatorname{RMSE}_{\mathrm{MCI}} & =\sqrt{\frac{\sum_{i=1}^{K}\left(I_{v}-\widehat{I}_{v i}\right)^{2}}{\left\|I_{v}\right\| K}} \times 100 \%,
\end{aligned}
$$

where $K$ is the number of Monte Carlo experiments and $N$ is the number of signals $\widehat{\theta}_{n, i} ; \widehat{\varphi}_{n, i}$ and $\theta_{n}, \varphi_{n}$ are the estimated value and true value by the $n$th signal and the $i$ th experiment, respectively; $\widehat{G}_{m i}, \widehat{P}_{m i}$ and $G_{m}, P_{m}$ are the estimated value and true value of gain and phase by the $m$ th sensor and the $i$ th experiment, respectively. In addition, $m=1, \ldots, q$; here $q=$ 5; $\widehat{R}_{v i}, \widehat{I}_{v i}$ and $R_{v}, I_{v}$ are the estimated value and true value of real part and imaginary part by the $v$ th mutual coupling coefficient and the $i$ th experiment, respectively; $v=1, \ldots, p-$ 1 ; here $p-1=2$.

4.1. DOA Estimation. In the first example, we present numerical simulation results to evaluate the superior performance of the proposed method in DOA estimation, in comparison with the gain and phase calibration method in $[17,20]$ and the mutual coupling calibration method in [10]. These methods are chosen because they are based on a similar data model where the auxiliary sensors are required, but these methods cannot calibrate gain and phase errors and mutual coupling errors simultaneously. Moreover, the results of MUSIC using the whole array with known uncertainties are also obtained. Assuming two signals from $-10^{\circ}$ and $10^{\circ}$, RMSEs of the DOA estimation are obtained by the above methods at different SNRs. 100 Monte Carlo experiments

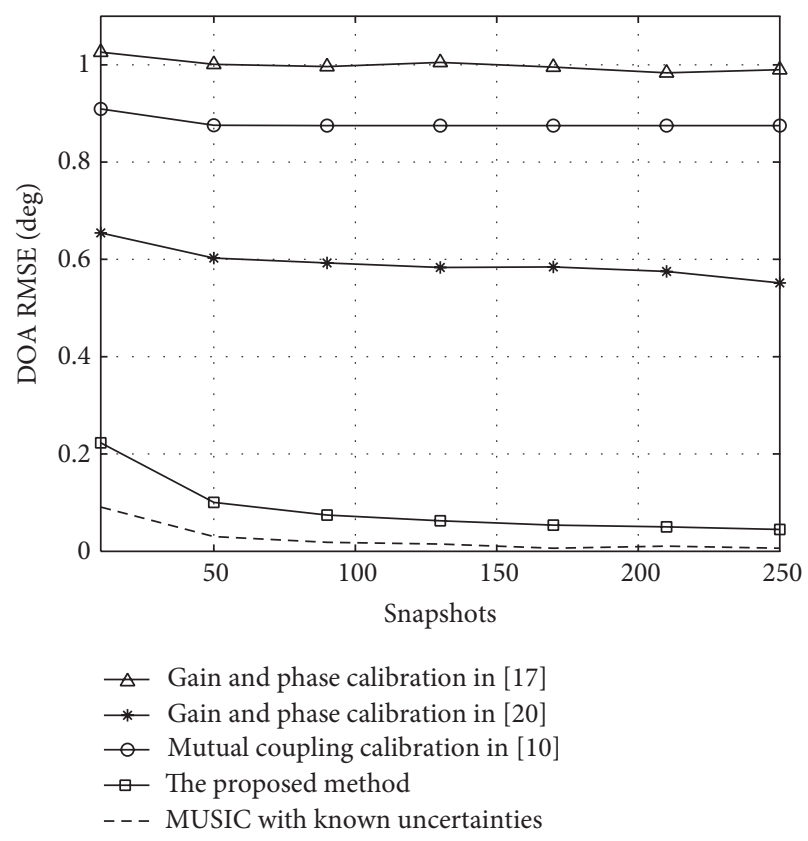

FIGURE 3: RMSE of two signals DOA estimation versus snapshot number.

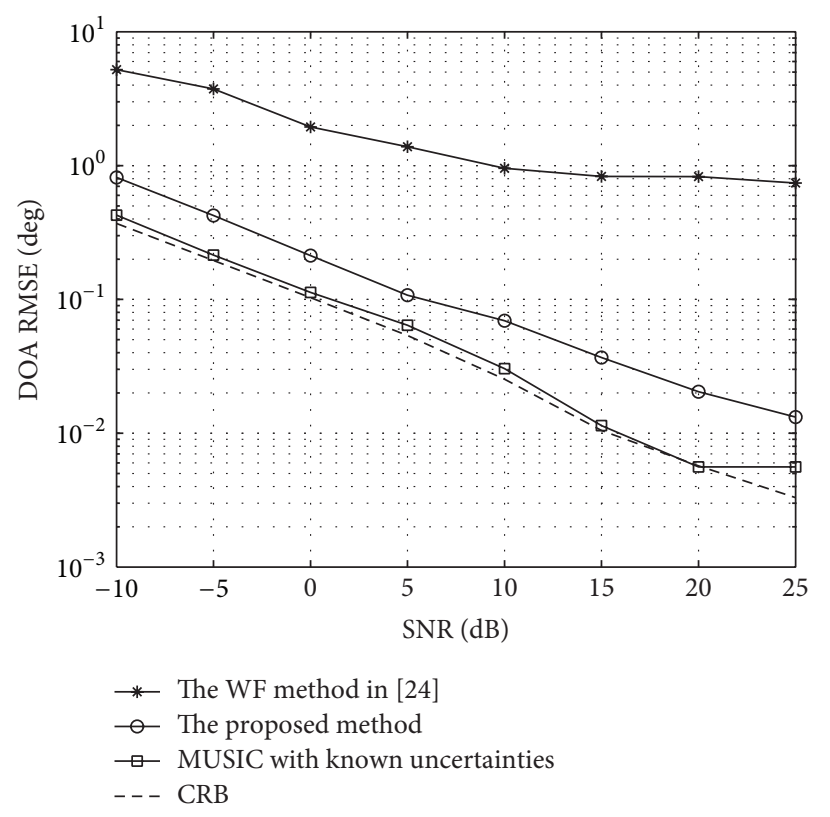

FIgURE 4: RMSE of three signals DOA estimation versus SNR.

are performed at each SNR, and in each experiment the snapshot number is 200 . The experiment results are shown in Figure 2. Figure 3 demonstrates the RMSE of the DOA estimation versus snapshot number with 100 Monte Carlo experiments, and the SNR is $10 \mathrm{~dB}$.

According to Figures 2 and 3, we can see that all methods work better as SNR and snapshot number increase. Generally speaking, the estimation performance will stabilize when SNR and snapshot number reach a certain threshold. 


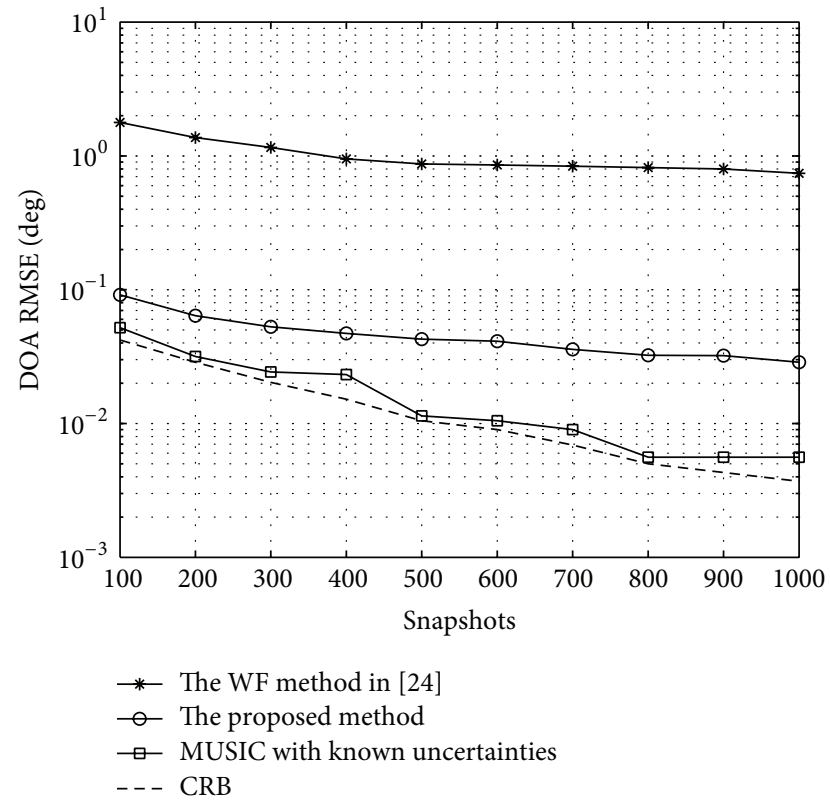

FigURE 5: RMSE of three signals DOA estimation versus snapshot number.

Moreover, it can be observed that the performance of the proposed method is close to the MUSIC algorithm with known uncertainties, which is much better than other methods. This is mainly because the proposed method can calibrate gain and phase errors as well as mutual coupling errors simultaneously while other methods can only calibrate one error, which seriously affects the performance of direction finding.

Next, performance comparison is made between the proposed method and the WF method which can calibrate these two types of errors at the same time through several experiments. The experiment results of MUSIC using the whole array with known uncertainties are also obtained, and CRB is displayed. Assuming three signals from $-10^{\circ}, 10^{\circ}$, and $20^{\circ}$, Figure 4 shows RMSE of DOA estimation versus SNR; 100 Monte Carlo experiments are performed at each SNR, and the snapshot number is 200; Figure 5 displays RMSE of the DOA estimation versus snapshot number with 100 Monte Carlo experiments, and the SNR is $10 \mathrm{~dB}$.

According to Figures 4 and 5, we can see that both methods work better as SNR and snapshot number increase, and the performance of the proposed method is better than that of the WF method. Moreover, the satisfactory performance of the proposed method can be achieved even at low SNRs and few snapshots. When SNR is less than $10 \mathrm{~dB}$, the performance of WF method deteriorates seriously while the proposed method is still effective. As snapshot number increases, the performance of WF method does not improve significantly. One possible explanation is that WF method is limited to the case where array errors are small, and its performance will be poor at low SNR. We can also note that the RMSE of DOA estimated by all methods except MUSIC with known uncertainties can not reach the CRB even at large SNRs and snapshot number.

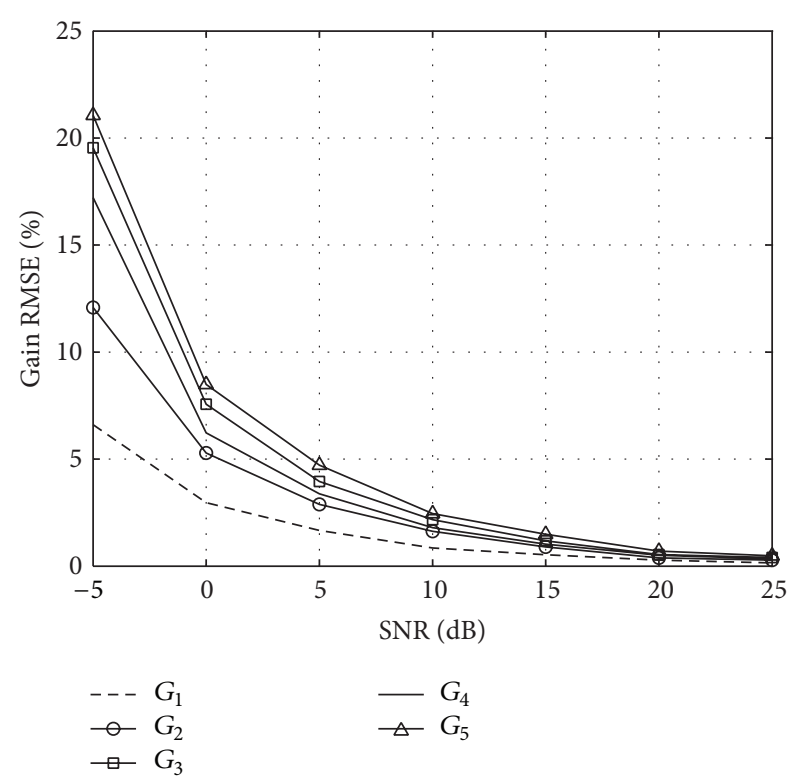

Figure 6: RMSE of gain coefficient estimation versus SNR.

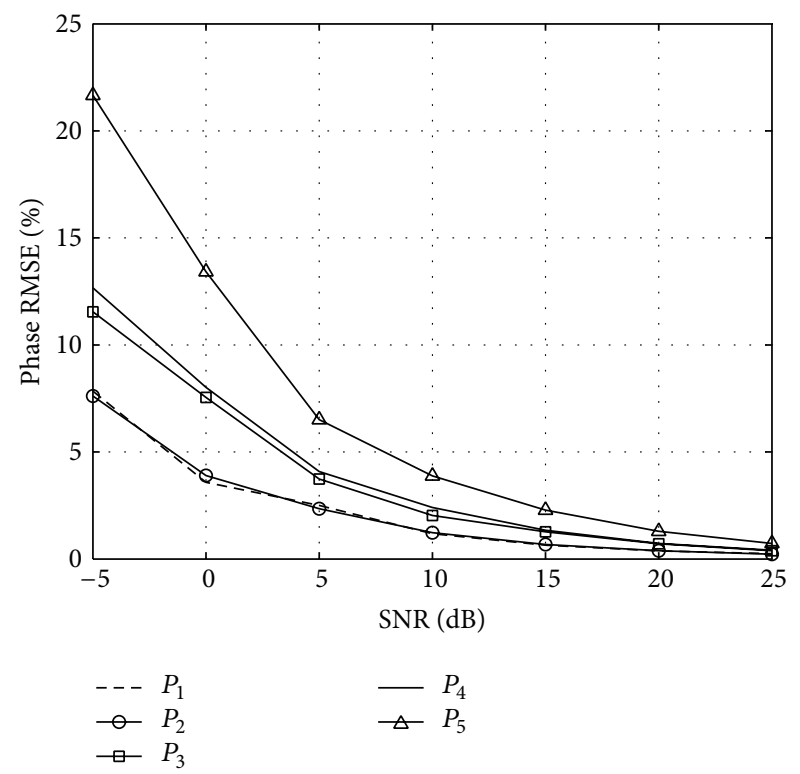

FIGURE 7: RMSE of phase coefficient estimation versus SNR.

4.2. Estimation of Gain and Phase Coefficients. Experimental results above have proved good performance of the proposed method for DOA estimation. The proposed method also can be used to estimate gain and phase coefficients. Assuming three signals from $-10^{\circ}, 10^{\circ}$, and $20^{\circ}$ the snapshot number is 200. In Figures 6 and 7, RMSE ${ }_{G}$ and $\mathrm{RMSE}_{P}$ at different SNRs from 100 Monte Carlo experiments are demonstrated, where $G_{m}, P_{m}, m=1, \ldots, 5$ represent gain and phase of each uncertain sensor. Table 1 shows mean and variance of gain and phase coefficients for all uncertain sensors at $\mathrm{SNR}=0 \mathrm{~dB}$ and $\mathrm{SNR}=20 \mathrm{~dB}$. 
TABLE 1: Mean and variance of gain and phase coefficients for all uncertain sensors at $\mathrm{SNR}=0 \mathrm{~dB}$ and $\mathrm{SNR}=20 \mathrm{~dB}$. True values are $G_{1}=0.80$, $G_{2}=1.25, G_{3}=1.53, G_{4}=0.75$, and $G_{5}=1.36 ; P_{1}=0.6283, P_{2}=-1.0472, P_{3}=-0.6283, P_{4}=0.7854$, and $P_{5}=-0.3142(\mathrm{rad})$.

\begin{tabular}{lcccccccccc}
\hline SNR & \multicolumn{9}{c}{$0 \mathrm{~dB}$} & \multicolumn{5}{c}{$20 \mathrm{~dB}$} \\
$m$ & 1 & 2 & 3 & 4 & 5 & 1 & 2 & 3 & 4 & 5 \\
\hline$\widehat{\widehat{G}}_{m}$ & 0.8087 & 1.2690 & 1.5651 & 0.7702 & 1.3699 & 0.7998 & 1.2504 & 1.5308 & 0.7504 & 1.3600 \\
$\sigma_{\widehat{\mathrm{G}}_{m}}$ & $8.2329 e-4$ & $3.8839 e-3$ & $1.0102 e-2$ & $3.2510 e-3$ & $1.2677 e-2$ & $8.0452 e-5$ & $1.5937 e-4$ & $3.0094 e-4$ & $5.0529 e-4$ & $4.8451 e-4$ \\
$\widehat{\widehat{P}}_{m}$ & 0.6316 & -1.0510 & -0.6257 & 0.7878 & -0.3139 & 0.6284 & -1.0470 & -0.6282 & 0.7854 & -0.3137 \\
$\sigma_{\widehat{P}_{m}}$ & $7.2978 e-5$ & $3.2913 e-5$ & $8.4479 e-5$ & $2.8760 e-5$ & $3.0745 e-5$ & $9.0441 e-6$ & $1.5761 e-5$ & $2.4466 e-5$ & $4.0881 e-5$ & $4.2990 e-6$ \\
\hline
\end{tabular}

TABLE 2: Mean and variance of the real and imaginary part of all mutual coupling coefficients at different SNRs. True values are $R_{1}=0.4499$, $R_{2}=0.2598, I_{1}=0.5362$, and $I_{2}=-0.1500$.

\begin{tabular}{lcccccccc}
\hline SNR & \multicolumn{2}{c}{$-5 \mathrm{~dB}$} & \multicolumn{2}{c}{$5 \mathrm{~dB}$} & \multicolumn{2}{c}{$15 \mathrm{~dB}$} & \multicolumn{2}{c}{$20 \mathrm{~dB}$} \\
$v$ & 1 & 2 & 1 & 2 & 1 & 2 & 0.2598 \\
$\overline{\widehat{R}}_{v}$ & 0.1387 & 0.2266 & 0.4189 & 0.2599 & 0.4459 & 0.2603 & 0.4497 \\
$\sigma_{\overline{\widehat{R}}_{v}}$ & $7.4330 e-3$ & $2.7175 e-3$ & $2.2405 e-3$ & $4.7373 e-4$ & $5.8136 e-4$ & $5.4347 e-5$ & $1.6664 e-5$ & $3.8597 e-6$ \\
$\overline{\widehat{I}}_{v}$ & 0.3669 & -0.1058 & 0.5185 & -0.1432 & 0.5344 & -0.1492 & 0.5359 & -0.1500 \\
$\sigma_{\bar{I}_{v}}$ & $8.6657 e-3$ & $1.2841 e-3$ & $1.1336 e-3$ & $1.3086 e-4$ & $7.3215 e-5$ & $1.6873 e-5$ & $1.0036 e-5$ & $1.8215 e-6$ \\
\hline
\end{tabular}

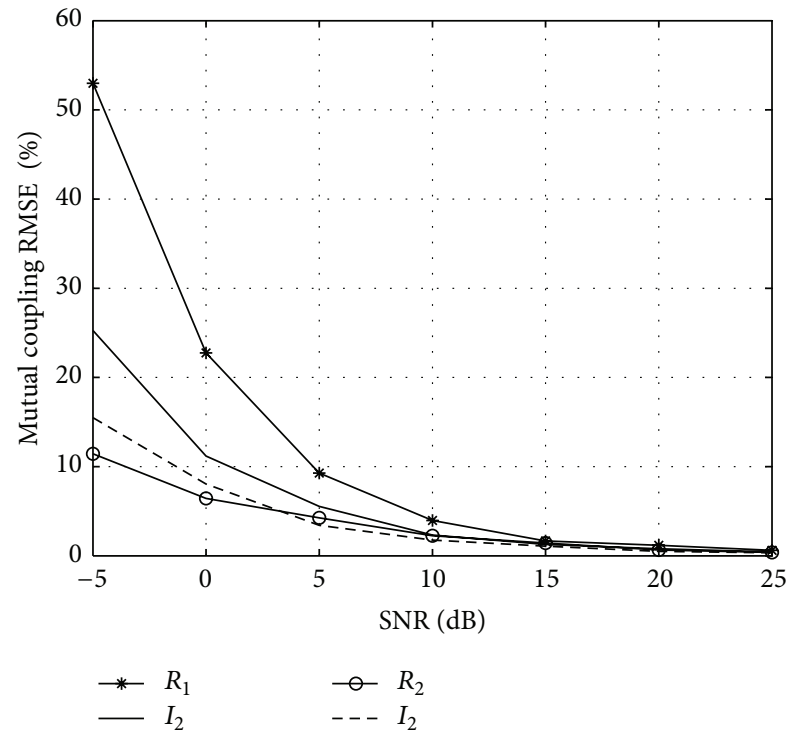

FIGURE 8: RMSE of mutual coupling coefficients versus SNR.

The experimental results show that the proposed method has a better performance in estimation of gain and phase coefficients as SNR increases. When SNR is higher than $5 \mathrm{~dB}$, RMSEs of gain and phase can be less than $10 \%$, which proves that when SNR is high enough, the coefficients could be estimated, with higher accuracy and stability.

4.3. Estimation of Mutual Coupling Coefficients. In the following experiments, the statistical efficiency of the proposed method in mutual coupling coefficient estimation is studied. Assuming three signals from $-10^{\circ}, 10^{\circ}$, and $20^{\circ}$ the snapshot number is 200. Figure 8 shows RMSE of real and imaginary part of mutual coupling coefficients $\mathrm{RMSE}_{\mathrm{MCR}}$ and RMSE $\mathrm{MCI}_{\mathrm{MC}}$ versus SNR. 100 Monte Carlo experiments are performed at each SNR. $R_{v}, I_{v}, v=1,2$ represent the real and imaginary part of each mutual coupling coefficient. Table 2 shows mean and variance of the real and imaginary part of all mutual coupling coefficients at different SNRs.

Figure 8 and Table 2 clearly illustrate that the proposed method has a better performance in estimation of mutual coupling coefficients as SNR increases. It is statistically efficient when SNR is higher than $5 \mathrm{~dB}$, and RMSEs are all less than $10 \%$.

In summary, simulation results have proved the effectiveness and excellent performance of the proposed method. As discussed in Section 4.1, better DOA estimation performance can be achieved when gain and phase errors and mutual coupling errors are simultaneously calibrated. Compared with the WF method, the satisfactory performance of the proposed method can be achieved even at low SNRs and few snapshots. As discussed in Sections 4.2 and 4.3, the proposed method can be used to accurately estimate gain and phase coefficients and mutual coupling coefficients, with even higher accuracy when SNR reaches a certain threshold.

\section{Conclusion}

In this paper, a simple and efficient method is proposed for direction finding in the presence of gain and phase errors as well as mutual coupling errors. By applying a group of auxiliary sensors, DOAs as well as gain and phase coefficients can be simultaneously estimated, and mutual coupling coefficients can also be estimated by utilizing the proposed decoupling method. Iterative operation, calibration sources, and spectral peak searching are not required for this method. However, auxiliary sensors are necessary to implement this algorithm, whose number affects algorithm performance. Different from other methods, the proposed method can achieve good performance with only a few auxiliary sensors. As shown in simulation results, the effectiveness and excellent 
performance of the proposed method are compared with other popular methods. DOA estimation can achieve good performance even at low SNRs and few snapshots. Moreover, gain and phase coefficients and mutual coupling coefficients can be accurately estimated when SNR reaches a certain threshold.

\section{Conflict of Interests}

The authors declare that there is no conflict of interests regarding the publication of this paper.

\section{Acknowledgments}

The authors thank the reviewers and editors for their corresponding contributions in making the paper more presentable. This work was supported in part by the National Science Foundation of China under Grant 61201410 and in part by the Fundamental Research Funds for the Central Universities under Grant no. HEUCF130804.

\section{References}

[1] H. Krim and M. Viberg, "Two decades of array signal processing research: the parametric approach," IEEE Signal Processing Magazine, vol. 13, no. 4, pp. 67-94, 1996.

[2] D. H. Johnson and D. E. Dudgeon, Array Signal ProcessingConcepts and Techniques, Prentice-Hall, Englewood Cliffs, NJ, USA, 1993.

[3] P. Stocia and R. Moses, Introduction to Spectral Analysis, Prentice-Hall, Upper Saddle River, NJ, USA, 2nd edition, 2004.

[4] S. Yin, S. Ding, A. Haghani, and H. Hao, "Data-driven monitoring for stochastic systems and its application on batch process," International Journal of Systems Science, vol. 44, no. 7, pp. 136611376, 2013.

[5] S. Yin, H. Luo, and S. Ding, "Real-time implementation of faulttolerant control systems with performance optimization," IEEE Transactions on Industrial Electronics, vol. 64, no. 5, pp. 24022411, 2014.

[6] R. O. Schmidt, "Multiple emitter location and signal parameter estimation," IEEE Transactions on Antennas and Propagation, vol. 34, no. 3, pp. 276-280, 1986.

[7] R. Roy, A. Paulraj, and T. Kailath, "ESPRIT—a subspace rotation approach to estimation of parameters of cisoids in noise," IEEE Transactions on Acoustics, Speech, and Signal Processing, vol. 34, no. 5, pp. 1340-1342, 1986.

[8] A. J. Weiss and B. Friedlander, "Effects of modeling errors on the resolution threshold of the music algorithm," IEEE Transactions on Signal Processing, vol. 42, no. 6, pp. 1519-1526, 1994.

[9] Y. Li and M. H. Er, "Theoretical analyses of gain and phase error calibration with optimal implementation for linear equispaced array," IEEE Transactions on Signal Processing, vol. 54, no. 2, pp. 712-723, 2006.

[10] K. C. Ho and L. Yang, "On the use of a calibration emitter for source localization in the presence of sensor position uncertainty," IEEE Transactions on Signal Processing, vol. 56, no. 12, pp. 5758-5772, 2008.

[11] B. C. Ng and C. M. Samson, "Sensor-array calibration using a maximum-likelihood approach," IEEE Transactions on Antennas and Propagation, vol. 44, no. 6, pp. 827-835, 1996.
[12] F. Sellone and A. Serra, "A novel online mutual coupling compensation algorithm for uniform and linear arrays," IEEE Transactions on Signal Processing, vol. 55, no. 2, pp. 560-573, 2007.

[13] C. Qi, Z. Chen, Y. Zhang, and Y. Wang, "DOA estimation and self-calibration algorithm for multiple subarrays in the presence of mutual coupling," IEE Proceedings-Radar, Sonar and Navigation, vol. 153, no. 4, pp. 333-337, 2006.

[14] L. Bin, G. C. Zhi, and C. Shing, "DOA estimation and tracking of ULAs with mutual coupling," IEEE Transactions on Aerospace and Electronic Systems, vol. 48, no. 1, pp. 891-905, 2012.

[15] Z. Ye and C. Liu, "On the resiliency of MUSIC direction finding against antenna sensor coupling," IEEE Transactions on Antennas and Propagation, vol. 56, no. 2, pp. 371-380, 2008.

[16] Z. Ye and C. Liu, "2-D DOA estimation in the presence of mutual coupling," IEEE Transactions on Antennas and Propagation, vol. 56, no. 10, pp. 3150-3158, 2008.

[17] L. Hong-Bing, G. Yi-Duo, G. Jian, and J. Jun, "Mutual coupling self-calibration algorithm for uniform linear array based on ESPRIT," in Proceedings of the 2nd International Conference on Consumer Electronics, Communications and Networks (CECNet '12), pp. 3323-3326, Yichang, China.

[18] Z. Ye, J. Dai, X. Xu, and X. Wu, "DOA estimation for uniform linear array with mutual coupling," IEEE Transactions on Aerospace and Electronic Systems, vol. 45, no. 1, pp. 280-288, 2009.

[19] C. Soon, L. Tong, Y. F. Huang, and R. Liu, "Subspace method for estimating sensor gains and phases," IEEE Transactions on Signal Processing, vol. 42, no. 4, pp. 973-976, 1994.

[20] A. Liu, G. Liao, C. Zeng, Z. Yang, and Q. Xu, "An eigenstructure method for estimating doa and sensor gain-phase errors," IEEE Transactions on Signal Processing, vol. 59, no. 12, pp. 5944-5956, 2011.

[21] C. Shenghong, Y. Zhongfu, X. Dongyang, and X. Xu, "A Hadamard product based method for DOA estimation and gain-phase error calibration," IEEE Transactions on Aerospace and Electronic Systems, vol. 49, no. 2, pp. 1224-11233, 2013.

[22] M. Pesavento, A. B. Gershman, and K. M. Wong, "Direction finding in partly calibrated sensor arrays composed of multiple subarrays," IEEE Transactions on Signal Processing, vol. 50, no. 9, pp. 2103-2115, 2002.

[23] C. M. S. See and A. B. Gershman, "Direction-of-arrival estimation in partly calibrated subarray-based sensor arrays," IEEE Transactions on Signal Processing, vol. 52, no. 2, pp. 329-338, 2004.

[24] S. A. Elkader, A. B. Gershman, and K. M. Wong, "Rank reduction direction-of-arrival estimators with an improved robustness against subarray orientation errors," IEEE Transactions on Signal Processing, vol. 54, no. 5, pp. 1951-1955, 2006.

[25] L. Bin and C. Shing-Chow, "Direction finding with partly calibrated uniform linear arrays," IEEE Transactions on Antennas and Propagation, vol. 60, no. 2, pp. 922-929, 2012.

[26] C. M. S. See, "Sensor array calibration in the presence of mutual coupling and unknown sensor gains and phases," Electronics Letters, vol. 30, no. 5, pp. 373-374, 1994.

[27] K. V. Stavropoulos and A. Manikas, "Array calibration in the presence of unknown sensor characteristics and mutual coupling," in Proceedings of the European Signal Processing Conference (EUSIPCO '00), vol. 3, pp. 1417-1420, 2000.

[28] W. Ding, "Sensor array calibration in presence of mutual coupling and cain/phase errors by combining the spatial-domain 
and time-domain waveform information of the calibration sources," Circuits, Systems, and Signal Processing, vol. 32, no. 3, pp. 1257-1292, 2013.

[29] S. Yin, S. Ding, A. Haghani, H. Hao, and P. Zhang, "A comparison study of basic datadriven fault diagnosis and process monitoring methods on the benchmark Tennessee Eastman process," Journal of Process Control, vol. 22, no. 9, pp. 1567-1581, 2012.

[30] B. Friedlander and A. J. Weiss, "Direction finding in the presence of mutual coupling," IEEE Transactions on Antennas and Propagation, vol. 39, no. 3, pp. 273-284, 1991.

[31] S. D. Solomon, D. A. Gray, Y. I. Abramovich, and S. J. Andersoni, "Receiver array calibration using disparate sources," IEEE Transactions on Antennas and Propagation, vol. 47, no. 3, pp. 496-505, 1999.

[32] S. Yin, G. Wang, and H. Karimi, "Data-driven design of robust fault detection system for wind turbines," Mechatronics, 2013.

[33] S. Yin, S. Ding, X. Xie, and H. Luo, "A review on basic datadriven approaches for industrial process monitoring," IEEE Transactions on Industrial Electronics, no. 99, 1 page, 2014.

[34] J. R. Magnus and H. Neudecker, "Mathematical preliminaries," in Matrix Differential Calculus with Applications in Statistics and Econometrics, 2nd edition, 1999. 


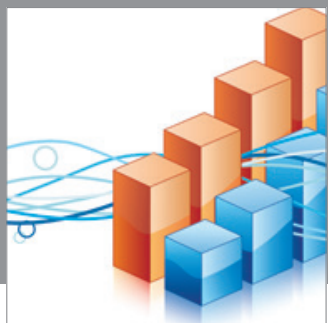

Advances in

Operations Research

mansans

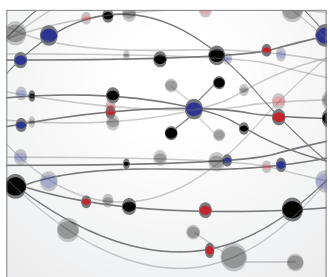

The Scientific World Journal
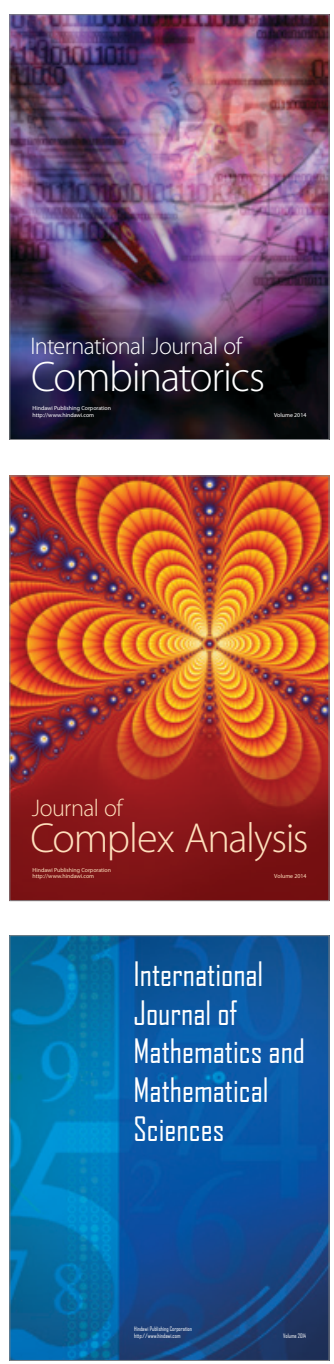
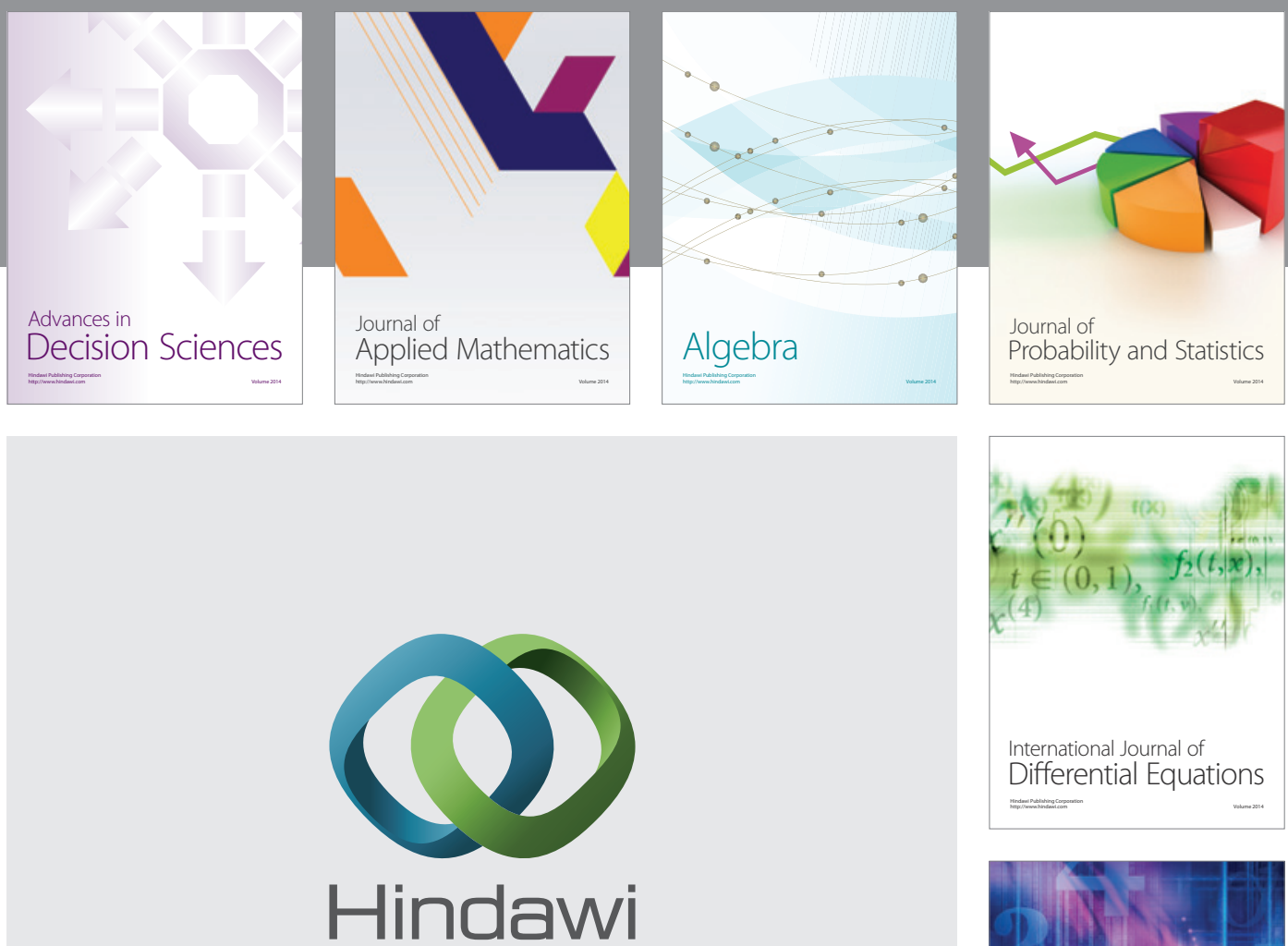

Submit your manuscripts at http://www.hindawi.com
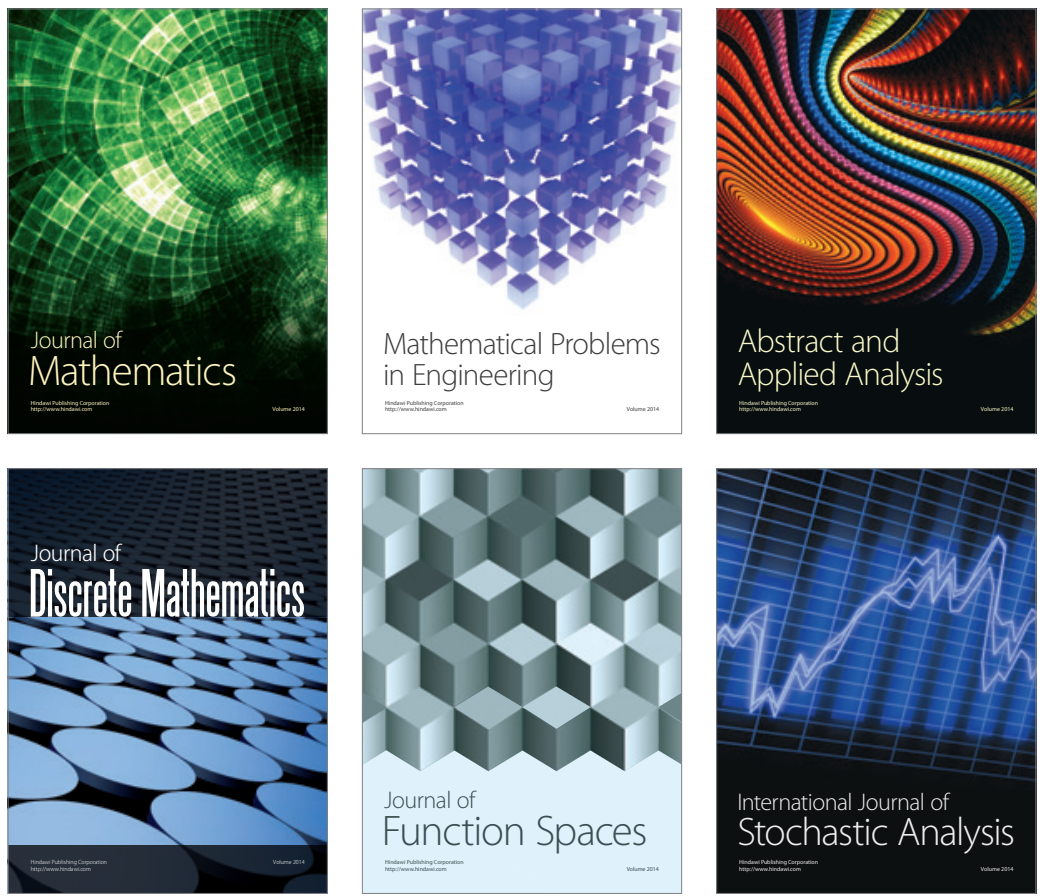

Journal of

Function Spaces

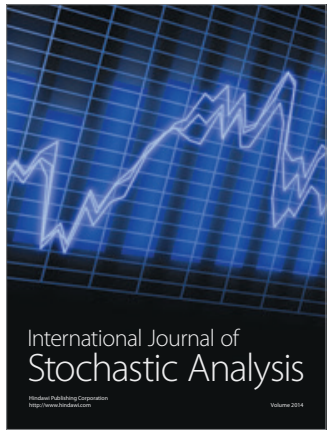

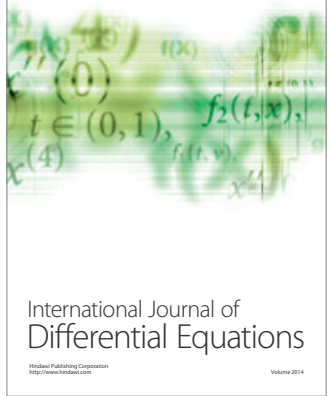
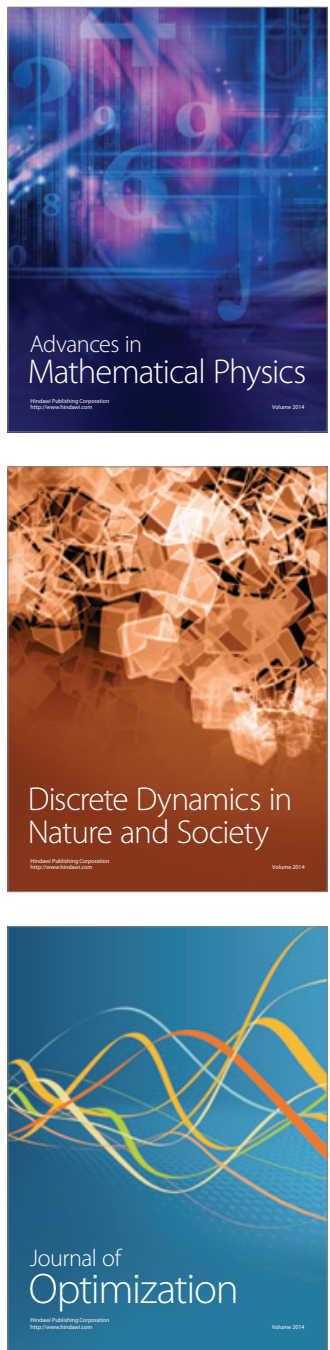